\title{
JAPAN ROUNDUP
}

Next April, Hitachi (Tokyo) plans commercial introduction of the world's fastest system for reading DNA sequencing gels. The automatic process allows determination of DNA sequences 5-10-times faster than manual methods currently in use: 200 nucleotides can be accurately determined in 3 to 4 minutes, according to Hitachi scientists. The system uses an argon laser to automatically determine positions in polyacrylamide sequencing gels of DNA fragments that have been tagged with a fluorescent dye. Similar argon laser devices have been developed by American companies, but Hitachi claims that its analyzer operates more than twice as fast as the U.S. competition.

Scientists at the Nagasaki Prefecture Marine Research Institute have genetically manipulated flounder eggs in vitro to produce a triploid strain that grows 40 percent faster than native fish. Marine Research Institute researchers speculate that the inability of the new strain to reproduce allows the utilization of those energy resources for faster growth. Work on mass production of these fish is currently in progress.

Researchers at Ube Industries (Tokyo) and in the Pharmacology Department of the Tokyo College of Science have developed a sensitive enzyme immunoassay kit for detecting aflatoxin $B_{1}$. The kit uses a monoclonal antibody that specifically binds aflatoxin $B_{1}$ to generate a color change in direct proportion to the amount of toxin in the sample. The kit became available for sale in July at a price of approximately $\$ 345$ for 80 tests.

Scientists at the Kyoto Pasteur Research Foundation have devised a screening procedure for the early detection of liver cancer. By detecting the decreased ability of infected blood cells to produce alpha- and gamma-interferon, the screening procedure is also useful for the early detection of hepatitis and cirrhosis of the liver. The reliability of the assay is currently under examination through clinical testing at 10 Japanese hospitals.

Scientists at Nippon Shokuzai Kagyo (Osaka), working in collaboration with colleagues at the Agricultural School at Miyazaki University, have used protoplast fusion to produce a novel hybrid mushroom. The new hybrid was obtained by fusing protoplasts derived from two species of the agratic (tree) mushroom hiratake (Pleurotus ostreatus): the Japanese tamogi-take and a species of hiratake that is native to Scotland. The taste and consistency of the new hybrid was reported to be excellent. Nippon Shokuzai Kagyo anticipates that the new mushroom will be widely used in cooking and will quickly gain acceptance in the marketplace.

Prepared by Hal Plotkin and Ken Coleman, Biotechnology in Japan Newsservice, Japan Pacific Associates (Palo Alto, $C A)$.

\section{PICO-INJECTOR}
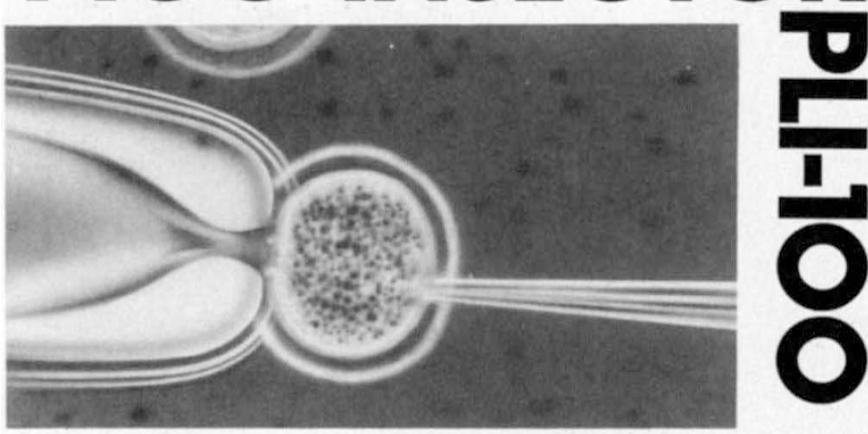

for all your injection needs... extracellular-intracellular

- Reproducible delivery • Digital displays • Balance/clear pressures - Vacuum fill/hold • Optional foot switches • Injection count
The Medical Systems Corporation PICOINJECTOR is a highly versatile single channel

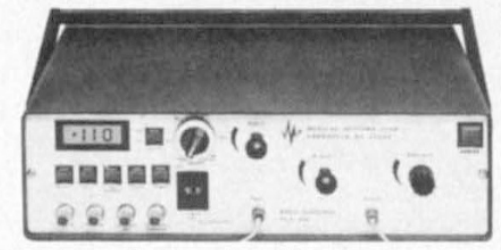

pressure unit that delivers volume from microliters to femtoliters using micropipettes. Its reproducible delivery allows a beginning or experienced user to master all aspects of microinjection quickly.

\section{THE RIGHT TECHNOLOGY

Call

\section{is the only journal to give you the news every month.}

Enter your subscription today. 